\title{
RIGIDITY THEOREMS FOR SURFACES IN EUCLIDEAN SPACE
}

\author{
BY BANG-YEN CHEN AND GERALD D. LUDDEN
}

Communicated by Philip Hartman, August 2, 1971

Let $M$ be a surface immersed in euclidean $m$-space $E^{m}$, and let $\nabla$ and $\nabla^{\prime}$ be the covariant differentiations of $M$ and $E^{m}$ respectively. Let $\boldsymbol{u}$ and $\boldsymbol{v}$ be two tangent vector fields on $M$. Then the second fundamental form $\boldsymbol{h}$ is given by

$$
\nabla_{u}^{\prime} v=\nabla_{u} v+h(u, v)
$$

If $e_{1}, e_{2}, e_{3}, \ldots, e_{m}$ is a local field of orthonormal frame such that $e_{1}, e_{2}$ are tangent to $M$ and $e_{3}, \ldots, e_{m}$ are normal to $M$, then the mean curvature vector $\boldsymbol{H}$ is given by

$$
H=\frac{1}{2} \sum_{i=1}^{2} h\left(e_{i}, e_{i}\right)
$$

For a normal vector field $\boldsymbol{\eta}$ and a tangent vector field $\boldsymbol{u}$ on $M$, let $\nabla_{\boldsymbol{u}}^{*} \boldsymbol{\eta}$ denote the normal component of $\nabla_{u}^{\prime} \eta$. Then $\nabla^{*}$ defines a connection in the normal bundle of $M$ in $E^{m}$. A normal vector field $\boldsymbol{\eta}$ is said to be parallel in the normal bundle if $\nabla^{*} \eta=0$. Let $h_{i j}^{r}, i, j=1,2, r=3, \ldots, m$, be the coefficients of the second fundamental form $\boldsymbol{h}$. Then the Gauss curvature $K$ and the normal curvature $K_{N}$ are given by

$$
\begin{gathered}
K=\sum_{r=3}^{m}\left(h_{11}^{r} h_{22}^{r}-h_{12}^{r} h_{12}^{r}\right), \\
K_{N}=\sum_{r, s=3}^{m}\left[\sum_{k=1}^{2}\left(h_{1 k}^{r} h_{2 k}^{s}-h_{2 k}^{r} h_{1 k}^{s}\right)\right]^{2},
\end{gathered}
$$

respectively. The mean curvature vector $\boldsymbol{H}$, the Gauss curvature $K$, and the normal curvature $K_{N}$ play important roles, in differential geometry, for surfaces in euclidean space.

Let $\langle$,$\rangle denote the scalar product of E^{m}$. If the mean curvature vector $\boldsymbol{H}$ is nowhere zero and there exists a function $f$ on $M$ such that $\langle\boldsymbol{h}(\boldsymbol{u}, \boldsymbol{v}), \boldsymbol{H}\rangle$ $=f\langle\boldsymbol{u}, \boldsymbol{v}\rangle$ for all tangent vector fields $\boldsymbol{u}, \boldsymbol{v}$ on $M$, then $M$ is called a pseudoumbilical surface of $E^{m}$.

AMS 1970 subject classifications. Primary 53A05, 53B25, 53C40.

Key words and phrases. Mean curvature vector, parallel in the normal bundle, Gauss curvature, normal curvature, circular cylinder, Veronese surface. 
We shall now define the Veronese surface. Let $(x, y, z)$ be the natural coordinate system in $E^{3}$ and $\left(u^{1}, u^{2}, u^{3}, u^{4}, u^{5}\right)$ the natural coordinate system in $E^{5}$. We consider a surface in $E^{5}$ given by

$$
\begin{gathered}
\left(\frac{c}{\sqrt{ } 3} x y, \frac{c}{\sqrt{ } 3} y z, \frac{c}{\sqrt{ } 3} x z, \frac{c}{2 \sqrt{ } 3}\left(x^{2}-y^{2}\right), \frac{c}{6}\left(x^{2}+y^{2}-2 z^{2}\right)\right), \\
x^{2}+y^{2}+z^{2}=3, \quad c=\text { constant }>0 .
\end{gathered}
$$

This surface is a real projective plane in $E^{5}$ with $\nabla^{*} \boldsymbol{H}=0, K=\frac{1}{3} c^{2}$ and $K_{N}=\frac{8}{9} c^{4}$. It is called the Veronese surface.

The main purpose of this paper is to announce two rigidity theorems for surfaces in euclidean space with mean curvature vector parallel in the normal bundle. Details will appear in [2].

THEOREM 1. The minimal surfaces of a hypersphere of $E^{m}$, the open pieces of a product space of two plane circles and the open pieces of a circular cylinder in $E^{3}$ are the only nonminimal surfaces in euclidean space with constant Gauss curvature and mean curvature vector parallel in the normal bundle.

The proof of this theorem is based on the following lemmas.

LEMMA 1. Let $M$ be a nonminimal surface in $E^{m}$ with $\nabla^{*} \boldsymbol{H}=0$. Then $M=M_{1} \cup M_{2}$, where $M_{1}$ is pseudo-umbilical and $K_{N}=0$ on $M_{2}$.

LEMMA 2. Let $M$ be a nonminimal surface in $E^{m}$ with $\nabla^{*} \boldsymbol{H}=0, K_{N}=0$ and $K=$ constant, then $K \geqq 0$.

LEMMA 3 [3]. If $M$ is a nonminimal surface in $E^{m}$ with $K \geqq 0 K_{N}=0$ and $\nabla^{*} \boldsymbol{H}=0$, then $M$ is one of the following surfaces; (1) a minimal surface of a hypersphere of $E^{m},(2)$ an open piece of a circular cylinder in $E^{3}$, or (3) an open piece of a product space of two plane circles.

For the normal curvature $K_{N}$, we have

THEOREM 2. The Veronese surface in $E^{5}$ is the only closed surface in $E^{m}$ with constant normal curvature $K_{N} \neq 0$ and $\nabla^{*} \boldsymbol{H}=0$.

The proof of Theorem 2 is based on Lemma 1 and a result of [1] which states that the Veronese surface is the only closed pseudo-umbilical surface in euclidean space with constant normal curvature $K_{N} \neq 0$.

\section{REFERENCES}

1. B.-Y. Chen, Pseudo-umbilical submanifolds of a Riemannian manifold of constant curvature. III,J. Differential Geometry (to appear).

2. B.-Y. Chen and G. D. Ludden, Surfaces with mean curvature vector parallel in the normal bundle (to appear).

3. J. A. Erbacher, Isometric immersions with constant mean curvature and triviality of the normal bundle, Nagoya Math. J. (to appear).

Department of Mathematics, Michigan State University, East Lansing, Michigan 48823 